\title{
TECNOLOGÍAS DISRUPTIVAS Y FLEXIBILIDAD CURRICULAR EN MAESTRÍAS DE MERCADOTECNIA 2019-2020
}

\author{
DISRUPTIVE TECHNOLOGIES AND CURRICULAR FLEXIBILITY IN MARKETING \\ MASTERS 2019-2020
}

\author{
Gabriela Velasco-Rodríguez ${ }^{1}$ * (D); Claudia Ivet Montellano-Rosas ${ }^{2}$ (D); \\ Sheyla Karina Vargas-Rosas ${ }^{2}$ (D). \\ 1.Universidad Autónoma de Ciudad Juárez. México.gvelasco@uacj.mx \\ 2.Universidad Autónoma de Ciudad Juárez. México. al156217@alumnos.uacj.mx \\ *Autor de Correspondencia: María de Lourdes Jiménez-Calvo, correo electrónico:maria.jimenezca@utn.edu.mx
}

\section{RESUMEN}

Existen una diversidad de modalidades y sistemas educativos en los planes y programas de estudio en las maestrías de mercadotecnia ante las tecnologías disruptivas. En este sentido, el objetivo general es describir las características claves de los planes de estudios en maestrías de mercadotecnia 2019-2020 ante las tecnologías disruptivas, desde un diseño de investigación descriptiva con base a diez aspectos ubicación, institución, nombre del programa, perfil de ingreso, perfil de egreso, estructura curricular, campo de orientación, orientación didáctica, idioma (en que se imparten las clases) y duración. Los resultados muestran que las principales características son: estructura curricular y orientación didáctica. Por lo tanto, se recomienda considerar dichos aspectos para lograr una flexibilidad curricular que responda al contexto actual.

Palabras clave: Tecnologías disruptivas; flexibilidad curricular; maestrías en mercadotecnia.

Cómo citar:

Velasco-Rodríguez, Gabriela; Montellano-Rosas, Claudia Ivet; Vargas-Rosas, Sheyla Karina. (2021). TECNOLOGÍAS DISRUPTIVAS Y FLEXIBILIDAD CURRICULAR EN MAESTRÍAS DE MERCADOTECNIA 2019-2020. Revista de Investigaciones Universidad del Quindio, 33(S1), 163-173. https://doi.org/10.33975/riuq.vol33nS1.490 


\begin{abstract}
There is a diversity of educational modalities and systems in the study plans and programs in marketing master's degrees in the face of disruptive technologies. In this sense, the general objective is to describe the key characteristics of the study plans in marketing master's degrees 2019-2020 in the face of disruptive technologies, from a descriptive research design based on ten aspects: location, institution, name of the program, profile of admission, graduation profile, curricular structure, field of orientation, didactic orientation, language (in which classes are taught) and duration. The results show that the main characteristics are: curricular structure and didactic orientation. Therefore, it is recommended to consider these aspects to achieve a curricular flexibility that responds to the current context.
\end{abstract}

Keywords: Disruptive technologies; curricular flexibility; master's degrees in marketing

\title{
INTRODUCCIÓN
}

\section{Antecedentes}

A lo largo del tiempo, han surgido transformaciones en la manera en que los países comercializan sus productos con otros países o incluso en su misma entidad, y el marketing es una herramienta de la cual se apoyan toda clase de empresas para la promoción y gestión de productos.

A partir de lo que según Barroso \& Martín (1999) “el marketing debe entenderse más como un proceso interactivo de gestión que como una función, ya que la gestión orientada al mercado se construye mejor sobre la relación que sobre la transacción" (citado en Gómez Bayona , Arrubla Zapata , Aristizábal Valencia , \& Restrepo Rojas, 2018, pág. 345).

Por otra parte, un estudio de Gómez \& Uribe, (2016) el marketing ha tenido cambios respecto a no solo concentrar la atención en el producto sino en el cliente, anteriormente se le consideraba marketing 1.0. Después, una vez que las empresas tomaron consciencia de la importancia que tiene el cliente, comenzarón a tomar decisiones para la creación de nuevas estrategias enfocándose en el comprador, y así surgió el marketing 2.0. En la actualidad, el marketing se ha renovado en la dinámica del mercado, en el cual las corporaciones prestan más atención en temas más humanistas, donde las ganancias deben estar más en igualdad con la responsabilidad corporativa, además, Kotler et al., (2018) plantea lo que se conoce actualmente como mercadeo 3.0. (citado en Gómez Bayona, et al., 2018, pág. 345). Con base a lo anterior, las instituciones de educación superior han transformado sus programas de maestría considerando las necesidades, deseos y demandas de un contexto globalizado, a través de la realización de estudios. De ahí, que se retoman los siguientes estudios que examinan distintos elementos en el nivel educativo de maestría.

Para empezar, Sepúlveda Cervantes (2015) desarrolló un estudio comparativo del centro Universitario de Ciencias Económico Administrativas (cucea), mediante un análisis de empleadores y egresados en la Maestría en Dirección de Mercadotecnia (MDM), del periodo de egresados 2006-2014. Retomando la investigación elaborada por la Agencia Corporativa de Servicios Integrales (ACSI, 2015). El objetivo principal fue comparar la percepción que tienen los egresados y empleadores con relación a las competencias de los egresados, considerando la valoración de habilidades e intelecto en el campo de acción. 
Por otro lado, Caro Lazos, Sánchez Acosta, \& Flores Morales (2017) ejecutaron un estudio de índole mixto, cuantitativo, comparativo y transversal, analizaron los modelos presentados uno por Sergio Tobón, el de las Universidades politécnicas y el de la Dirección de Investigación y Posgrado de la U.ACH en un nivel de maestría. La muestra estuvo conformada por egresados en 2013, de las maestrías en administración, administración de recursos humanos, mercadotecnia y sistemas de información, los docentes pertenecientes al programa, y los empleadores con un nivel gerencial en distintas corporaciones en las cuales laboraron los egresados de los programas académicos.

Además, Gómez Bayona , Arrubla Zapata, Aristizábal Valencia , \& Restrepo Rojas (2018) realizaron un estudio de tipo exploratorio el cual tuvo como objetivo identificar el valor de las estrategias de marketing relacional en el sector académico, principalmente en las universidades de Colombia y España para saber de que forma aplican acciones mercadológicas. Con un método de revisión sistemática de literatura, encontraron los distintos cambios generacionales de los años del 2000 hasta 2018 en el concepto de marketing relacional. La población fue obtenida del ránking QS, en donde tomaron las 10 mejores universidades de España y Colombia, donde se consideran las diferentes estrategias de marketing relacional que usan para acercarse a sus grupos de interés.

También, Jiménez Moreno, Caso Niebla , \& Díaz López (2020), ejecutaron un estudio con un diseño transversal, con la finalidad de hacer un diagnóstico de 46 académicas de la universidad Autónoma de Baja California en sus 3 campus, ubicados en Mexicali, Tijuana y Ensenada. Se evaluaron dos esferas, las cuales fueron: competitividad académica y cobertura, y acreditación de programas educativos.

\section{Planteamiento del problema}

En el siglo XXI surge lo que se conoce como Industria 4.0 con la intención de llevar a cabo la "automatización de gran parte de la producción industrial, por medio de la interacción de la inteligencia artificial, el internet de las cosas, la biotecnología y los nuevos modelos de hacer negocios basados en el Big Data o el Blockchain (Garcés León , Millán Pelayo, Peña Pulido , \& Torres Belalcázar, 2020, pág. 2).

Es imprescindible que los próximos profesionales que se incorporen al área laboral deben contar con conocimientos sobre la Industria 4.0. Garcés León, et al., (2020), mencionan que requieren de competencias como las siguientes: "la creatividad, la utilización de la tecnología, el ejercicio del liderazgo social y empresarial, la utilización de la ciencia, la práctica de la investigación, la aplicación y respeto de la sostenibilidad ambiental y la vivencia de la ética" (pág.5).

Por otro lado, la crisis sanitaria (COVID-19) que se está presentando mundialmente, causo que las actividades de cualquier índole en su mayoría se deban realizar a través de plataformas virtuales, y no es la excepción del quehacer educativo superior, debido a esta situación, los establecimientos que ofrecen programas de maestría únicamente escolarizados han tenido que capacitarse y adaptarse a los nuevos sistemas educativos. Aunque, existen universidades que anteriormente ya brindaban los programas con un sistema de educación a distancia y semipresencial.

De todos estos hechos se desprenden que las demandas que surgen ante un mundo globalizado, son que las instituciones que ofrecen estudios de educación superior específicamente los de maestría en marketing, deben mejorar la calidad de los programas en cuanto al perfil de ingreso y egreso, asimismo la flexibilidad curricular que ofrecen a los estudiantes. 
No obstante, sino se atiende el problema de mejorar la calidad de los planes educativos es probable que los futuros egresados no cuenten con las competencias necesarias para tener un rendimiento satisfactorio ante los tendencias y retos de la industrialización ante un entorno global implicando que en el caso de Latinoamérica y en lo particular México sigan ofreciendo una baja calidad en sus programas educativos de maestría.

En tal sentido México a través de la Secretaria de Educación Pública (SEP) plantea que uno de los problemas públicos es que "las y los estudiantes no reciben una educación de calidad, relevante y pertinente en los diferentes tipos de niveles y modalidades del Sistema Educativo Nacional que favorezca su desarrollo humano integral" (Secretaría de Educación Pública SEP , 2020, págs. 201202). Por ello, México necesita de programas altamente competitivos para atender esta problemática, a parte que es un derecho de la ciudadanía poder tener acceso a una buena educación.

De ahí que han surgido investigaciones en el área académica y laboral con el objetivo de analizar las competencias de los egresados, la competitividad de las universidades que ofrecen programas de educación superior, y la manera en que los empleadores evalúan a los egresados que contratan.

Con base a lo anterior la presente investigación considera que una alternativa ante la problemática planteada es realizar en lo particular un diagnóstico sobre las características de los programas de las maestrías en marketing, es decir, analizar la oferta educativa vigente de las universidades internacionales, nacionales y locales con la finalidad de establecer los aspectos relevantes para el diseño curricular de una maestría en marketing que responda a la industrialización en el contexto global.

Con la finalidad de contar con egresados que posean las habilidades pertinentes para el cumplimiento del perfil que solicitan las empresas, asimismo esto implica contar con docentes comprometidos en adquirir las competencias necesarias para poder fomentar una enseñanza de calidad, de igual manera, es indispensable que los estudiantes tengan el gusto y el compromiso de aprender y desempeñarse en el área de interés.

\section{Objetivo de estudio}

Describir las características claves de los planes de estudios en maestrías de mercadotecnia 2019-2020 ante las tecnologías disruptivas.

\section{MARCO TEÓRICO}

En relación con la industria 4.0, el significado de este concepto surge en el siglo XXI en Alemania y lo relacionan al ámbito político económico en donde las tecnologías son necesarias para poder llevar a cabo planificaciones. Asimismo, se define como "una maquinaria física y dispositivos con sensores y software que trabajan en red y permiten predecir, controlar y planear mejor los negocios y los resultados organizacionales" ( Ynzunza Cortés, Izar Landeta, Bocarando Chacón, Aguilar Pereyra, \& Larios Osorio, 2017, pág. 34, como se citó en Ning \& Liu, 2015). Dicho esto, a futuro se espera un gran avance en donde las maquinas podrán lograr interactuar unas con otras, intercambiando información y realizando acciones (Ynzunza Cortés et al., 2017).

De acuerdo con Sánchez Gúzman (2019), la industria 4.0 esta conformada con lo siguiente: "herramientas, metodologías, recursos humanos innovadores y cambio en la manera de concebir a la 
industria... es una guía que servirá para señalar el o los caminos a seguir por los siguientes años, sin tener claro una etapa de duración" (pág.47). Igualmente, Garcés León, Millán Pelayo, Peña Pulido , y Torres Belalcázar (2020) mencionan que la industria 4.0 o cuarta revolución industrial esta orientada a las tecnología de uso físico y digital, siendo estas las que apoyan a las diferentes organizaciones a llevar a cabo innovaciones y el uso eficiente de recursos.

Según Franky vicerrector de la Universidad Nacional de Colombia menciona que: la cuarta revolución industrial está guiada por el desarrollo de conocimientos y, por ende, su base deben ser las instituciones educativas" (Sostenible, 2018, citado en Gaercés León, et al., 2020, pág.5). De esta manera, las escuelas deben comenzar a integrar estrategias para afrontar de manera efectiva este cambio, ya que, Jalil Naji (2018) refiere que el cambio de integraciones tecnológicas al ámbito laboral posiblemente llevara a la necesidad de desaparecer empleos, ya que no se necesitara de individuos para realizar las tareas diarias, sin embargo, menciona que surgirán otros nuevos.

En cuanto a la flexibilidad curricular, Pedroza (2000) expresa que "la flexibilidad curricular es la que plantea a la enseñanza como un binomio de ciencia-acción, como una forma de incentivar la práctica reflexiva del saber y el saber hacer" (citado en Romero y Gildo, 2012, pág. 287). Por su parte, Romero y Gildo (2012) observan que la flexibilidad debe ser tomada en cuenta a favor de las necesidades sociales y lo que estas demandan. Debido a que, la sociedad necesita de personas mayormente capacitadas para realizar acciones que produzcan nuevo conocimiento. También, exponen 4 aspectos claves a considerar en la flexibilidad curricular, como el método de aprendizaje, el lugar en donde se da el aprendizaje, elementos teóricos y de referencia, y la forma en que se integra el currículo.

A su vez, dos instituciones elaboran un plan donde se visibiliza la flexibilidad curricular en los planes de estudio de educación superior en México, la Asociación Nacional de Universidades e Instituciones de Educación Superior (ANUIES) con educación superior siglo XX y la Secretaría de Educación Pública (SEP) con el programa de Educación Superior 2001-2006. De igual modo, otros organismos han destacan la importancia de un currículo flexible, como lo son la Organización para Cooperación y el Desarrollo Económicos (OCDE), el Banco Mundial, y proyectos internacionales que han creado archivos como lo es el informe Atalli del año 2002 (Romero y Gildo, 2012).

\section{MÉTODO}

El diseño de investigación es descriptivo, donde se optó por una muestra por conveniencia de las maestrías en mercadotecnia consultando distintas fuentes de información en instituciones a nivel nacional e internacional.

\section{Técnica de recolección de datos}

En la recolección de datos se hace acopio de aquellos programas de maestría pertenecientes al área de mercadotecnia o afines a esta, que se obtiene de universidades, escuelas de negocios o institutos, nacionales e internacionales que cuenten con programas de maestría, magíster o MBA.

\section{Técnica de análisis e interpretación de datos}

Con base a una revisión de documentos y registros de datos cualitativos diversos permite conocer los elementos que caracterizan a los programas de maestría en marketing que se mencionan en los mismos, 
los cuales son útiles para realizar el análisis de la situación actual respecto al diseño curricular a nivel nacional e internacional en diferentes páginas de universidades, en el ránking de América Economía (América Economía, s.f.) e instituciones que regulan las maestrías a nivel nacional (CONACYT Consejo Nacional de Ciencia y Tecnología, 2019)

Para ello, se identifican los siguientes temas del diseño curricular de cada uno de los programas de maestría que son: ubicación, institución, nombre del programa, perfil de ingreso, perfil de egreso, estructura curricular, campo de orientación, orientación didáctica, idioma (en que se imparten las clases) y duración.

Ubicación. Especificar en qué país, ciudad o estado se encuentra ofertada la maestría.

Institución. Universidad, escuela o instituto que imparte la maestría.

Programa. Titulo o nombre de la maestría, desde este punto se especifica el enfoque que tiene la maestría.

Perfil de ingreso. Comprende los requisitos que el estudiante o postulante debe cumplir para ser considerado en la admisión a 1 maestría.

Perfil de egreso. Son los conocimientos, habilidades y valores que obtiene el egresado de la maestría. Plan curricular. Incluye la determinación de los contenidos curriculares, así como su organización y estructuración, es decir, carga académica que contienen los programas.

Campo de Orientación. Investigación o profesionalizante, en este caso, se seleccionó a la orientación profesionalizante.

Orientación didáctica. Se considera la modalidad del programa (presencial, semipresencial o en línea) que se traducen a los horarios en que son ofrecidos los cursos que comprenden la maestría, ya sea de tiempo parcial, tiempo completo, weekend o executive.

Idioma. Se trata de la incorporación del idioma tanto el procedimiento de ingreso (TOEFL), asimismo en la impartición de las clases.

Duración. Se refiere a la duración del programa que se presenta por meses, años u horas.

\section{HALLAZGOS}

Las características a nivel global que se observaron fue que los países con mayor oferta educativa son España y Chile. Además, las maestrías pueden identificarse bajo el nombre de mercadeo, mercadotecnia y marketing o la combinación de mercadotecnia con publicidad, gestión comercial o con dirección. Esto muestra que las maestrías de mercadotecnia están ligadas con administración, publicidad y área de ventas.

El perfil de ingreso de las maestrías está orientado a personas con experiencia laboral, aunque existen algunas que también están dirigidas a recién egresados. En lo particular a los que trabajan se les pide por lo menos de tres a cinco años de experiencia, así como el requisito del examen de TOEFL. La duración es de dos años o año y medio. Referente a la orientación didáctica para los estudiantes que trabajan se manejan distintos sistemas de clases como tiempo completo, medio tiempo, fines de semana, ejecutivo e hibrido.

Se observó que en España la institución ESIC Business \& Marketing School es una gran exponente de educación en mercadotécnica, ya que ofrece diversos programas relacionados a esta. Por otra parte, los ránkings de América Economía incluyen la escuela IE Business School (España) entre las mejores escuelas, de igual manera la universidad Adolfo Ibáñez, ESAN Graduate School of Business, 
Universidad de los Andes, Universidad de San Andrés y la Universidad de Chile, son pertenecientes a este ránking y ofrecen maestrías en marketing. Por lo tanto, se puede inferir que los programas que ofertan son de calidad y tienen una gran calificación. Además, Hult Busniess School se encuentra en este ránking, siendo una escuela de Estados Unidos como ESADE Business School.

A nivel nacional se identifican 169 maestrías en mercadotecnia y áreas afines en las seis zonas que conforman a México, una vez que se realizó la revisión del anuario de ANUIES 2019-2020

En la Zona Norte se encontró un total 32 maestrías, 2 (6.25\%), Baja California, 1 (3.12\%) Sonora, 4 (12.5\%) Chihuahua, 3 (9.37\%) Coahuila, 19 (59.37\%) Nuevo León y 3 (9.37) Tamaulipas. En la Zona Sureste se encontró un total 74 maestrías, 3 (4.05\%) Campeche, 9 (12.16\%) Chiapas, 2 (2.70\%) Guerrero, 6(8.1\%) Oaxaca, 24 (32.4\%) Puebla, 7(9.45\%) Quintana Roo, 3 (4.05\%) Tabasco, 12 (16.21\%) Veracruz y $8(10.81 \%)$ Yucatán.

En la Zona Noreste cuentan con un total 9 maestrías, 3 (33.3\%) Durango, 2 (22.22\%) Nayarit, 3 (33.3\%) Sinaloa y 1 (11.1\%) Zacatecas. En la Zona Bajío Occidente con un total de 24 maestrías, 3 (12.5\%) Aguascalientes, 1(4.1\%) Colima, 6 (25\%) Guanajuato, 4 (16.6\%) Jalisco, 4 (16.6\%) Michoacán, 4 (16.6\%) Querétaro y 2 (8.33) San Luis Potosí

En la Zona Ciudad de México y Metropolitana ofrecen un total de 30 maestrías, 21 (70\%) en Ciudad de México, 4 (13.3\%) en México, 3 (10\%) Hidalgo, 1 (3.3\%) Morelos y 1 (3.3\%) Tlaxcala. De tal manera que en la Zona Norte se concentra principalmente en el estado de Nuevo, en la zona sureste es el estado Puebla, en la zona noreste es Durango, en la zona del Bajío Occidente se encontró a Guanajuato y en la zona Ciudad de México y Metropolitana a la Ciudad de México.

Por otra parte, se consideraron los programas de maestrías en marketing como de mercadotecnia con orientación profesional reconocidos en el Padrón del Programa Nacional de Postgrados de Calidad en CONACYT. (CONACYT Consejo Nacional de Ciencia y Tecnología, 2019)

En cuanto a las maestrías que se tomaron en cuenta a nivel nacional pertenecen al PNCP de CONACYT, siendo cuatro provenientes de los estados de Nuevo León, Jalisco, Puebla y Sonora. En concreto, dos se llaman dirección y mercadotecnia, otra marketing y mercados de consumo y finalmente, maestría en administración en gestión de capital humano y estrategias de mercadotecnia.

El perfil de ingreso en la universidad de Puebla requiere comprobante de suficiencia del idioma ingles TOEFL mínimo 450 pts., y un examen psicométrico; en la universidad de Guadalajara se pide contar con abstracción mental; y la universidad de Sonora pide acreditación de examen TOEFL de al menos 481 pts. Por otro lado, el perfil de egreso expresa ser un líder, estratégico y analítico.

A su vez, las cuatro maestrías tienen modalidad escolarizada, la maestría en Dirección de Mercadotecnia en la Universidad de Guadalajara se contempla las modalidades de tiempo parcial ( $\sin$ apoyo de CONACYT) y tiempo completo (apoyo de CONACYT) para cursar 16 materias; la maestría en Administración con orientación en Gestión de Capital Humano y Estrategias de Mercadotecnia en la Universidad Autónoma de Nuevo León se presenta la modalidad: presencial/ semestral/modular: 2 módulos por 9 semanas por semestre, 2 materias en cada módulo, siendo 4 materias en total al semestre para cursar 16 materias en 2 años; y la maestría en Dirección y Mercadotecnia de la Universidad Popular Autónoma del Estado de Puebla, A.C. se muestra que 
su modalidad es presencial para cursar 12 materias. En la maestría en Marketing y Mercados de Consumo de la Universidad de Sonora para cursar 16 materias. Así mismo, tres maestrías tienen una duración de dos años aproximadamente.

\section{DISCUSIÓN/CONCLUSIÓN}

Ante las tecnologías disruptivas que son los medios de interacción de la inteligencia artificial, el internet de las cosas y robótica (Garcés León, Millán Pelayo, Peña Pulido , \& Torres Belalcázar, 2020, pág. 2), las instituciones que ofrecen maestrías en mercadotecnia requieren una adaptación constante en su orientación educativa. Es conveniente destacar que la orientación educativa abarca modalidad y sistema. La clasificación de modalidad educativa incluye escolarizado, no escolarizado o mixto (Consejo Nacional de Ciencia y Tecnología CONACYT, 2021), así como el sistema puede ser presencial, semipresencial o a distancia. Por todo lo anterior, la adaptación a las tecnologías disruptivas implica una flexibilidad curricular que se da en función de un sistema de enseñanza aprendizaje, el contexto institucional, como la disciplina, su marco teórico y metodologías de referencia, así como la implementación del currículo (Romero Ortiz \& Gildo de la Cruz, 2012, pág. 28).

De ahí que los programas son orientados a la personalización de los estudiantes respondiendo al campo laboral y a la competencia que es mucha, considerando las tantas y distintas escuelas que se encontraron en la investigación. El contexto globalizado está muy presente en las maestrías, ya que en los distintos programas se ofrecen. En base a los programas de estudio revisados en cuanto a las maestrías de mercadotecnia a nivel internacional y nacional, se puede tomar en cuenta varios factores para el desarrollo del posgrado o maestría ideal que se ajuste y cumpla con los requerimientos necesarios en cuanto a la cuarta revolución industrial.

Primero se puede considerar los planes de estudios o planes curriculares. Si bien la mercadotecnia es afín a la administración; lo que demanda el sistema educativo ante la cuarta revolución industrial es más concentración en la mercadotecnia digital y las bases de datos. Con esto se trata de decir que, si bien son importantes los temas de cómo administrar, los aspectos económicos y financieros deben ser un complemento y no el tema central.

La concentración que se busca es la de la perspectiva del consumidor ya que el entorno globalizado implica mayor competencia, no solamente a nivel nacional sino también a nivel internacional y esto deriva en una gran oferta para el consumidor, es por esto que los estudiantes o futuros profesionistas deben satisfacer las futuras necesidades de consumidores y estas están siendo orientadas en base al entorno digital, que abarca el internet, las ventas en línea y las redes sociales con una gran influencia en el sector formal como informal del comercio.

En cuanto a la estructura de los planes académicos deben ir enfocadas a la internacionalización, ya que la competencia en la educación es un tema que necesita ir al ámbito global, debido a que las empresas lo requieren. Por ello, debe haber una cierta flexibilidad a la hora de ver las cargas académicas, para así poder entrar en un amplio entorno y cubrir las necesidades no solo de los contratantes, sino también de los estudiantes que necesitan desarrollar conocimientos amplios a la hora de competir con otros profesionales. En relación con la modalidad de la impartición de clases, se ha experimentado una nueva manera de trabajar, de impartir o de tomar clases, dando como consecuencia una educación a distancia, teletrabajo o de home office, donde se acerca más al sistema de educación 4.0 que se basa en las principales tendencias de innovación y cambio centradas en las competencias del siglo XXI, 
tales como la autodirección, autoevaluación y el trabajo en equipo donde el aprendizaje se basa en proyectos con el uso de la tecnología.

Dicho lo anterior, una de las principales propuestas que se tienen, es empezar a incluir en los programas de estudio de las maestrías en mercadotecnia, materias relacionadas a las bases de datos y la inteligencia artificial como recursos para cumplir con los nuevos requerimientos de la industria 4.0. Cursos didácticos sobre cómo se utilizan ciertos programas o tecnologías con el fin de que los profesionales puedan manipular por si mismos dichos programas o máquinas. También, se sugiere el impartir clases en línea, ya que esto mejora la productividad y permite que se aproveche mejor el tiempo y se desarrollen capacidades en cuanto al uso de las tecnologías. El desarrollo de programas curriculares más centrados en estas áreas hará que los estudiantes desarrollen enfoques tecnológicos y sobre todo puedan tener las herramientas necesarias para competir en el ámbito laboral que es a un nivel global.

\section{REFERENCIAS}

1. Ynzunza Cortés, C. B., Izar Landeta, J. M., Bocarando Chacón, J. G., Aguilar Pereyra, F., \& Larios Osorio, M. (2017). El entorno de la Industria 4.0: Implicaciones y Perspectivas Futuras. Conciencia tecnológica(54), 33-45.

2. América Economía. (s.f.). Ránkings. Obtenido de América Economía: https://www.americaeconomia. com/ránkings

3. CONACYT Consejo Nacional de Ciencia y Tecnología. (2019). Padrón del Programa Nacional de Posgrados de Calidad. Recuperado el Febrero de 2021, de CONACYT Consejo Nacional de Ciencia y Tecnología: http://svrtmp.main.conacyt.mx/ConsultasPNPC/padron-pnpc.php

4. EOI Escuela de Organización Industrial. (s.f.). MBA full time más Especialidad Sevilla. Recuperado el Febrero de 2021, de EOI Escuela de Organización Industrial: https://www.eoi.es/es/cursos/16517/ mba-full-time-especialidad-sevilla?

5. Esade Ramon Llull University. (2021). Full time MBA. Recuperado el Febrero de 2021, de Esade Ramon Llull University: https:/www.esade.edu/mba/en/programmes/full-time-mba/admissions/ apply-for-admission

6. Esan Graduate School of Business. (2019). Maestría en Marketing. Recuperado el Febrero de 2021, de Esan Graduate School of Business: https://www.esan.edu.pe/maestrias/marketing/

7. ESAN Graduate school of Business. (2019). MBA Weekends. Recuperado el Febrero de 2021, de ESAN Graduate school of Business: https:/www.esan.edu.pe/mba/weekends/

8. Esan Graduate shcool of Business. (2019). MBA Tiempo Parcial. Recuperado el Febrero de 2021, de Esan Graduate shcool of Business: https:/www.esan.edu.pe/mba/tiempo-parcial/

9. Eseune Business School. (s.f.). Executive MBA con Presencialidad Digital. Recuperado el Febrero de 2021, de https:/www.eseune.edu/mba-presencialidad-digital-videoconferencia/

10. Eseune Business School. (s.f.). Master en Dirección de Empresas Global Executive MBA Online. Recuperado el Febrero de 2021, de Eseune Business School: https:/www.eseune.edu/mba-onlinezoom-3/

11. ESIC Business and Marketing School. (2020). Executive Maestría en Dirección de Marketing y Gestión Comercial. Recuperado el Febrero de 2021, de ESIC Business and Marketing School: https:/www.esic.edu/master-y-postgrado/master-en-direccion-de-marketing-y-gestion-comercialgesco\#badajoz

12. ESIC Business and Marketing School. (2020). Maestría en Dirección de Comunicación y Gestión Publicitaria. Recuperado el Febrero de 2021, de ESIC Business and Marketing School: https://www. esic.edu/master-y-postgrado/master-en-direccion-de-comunicacion-y-publicidad-mpc\#barcelona

13. ESIC Business and Marketing School. (2020). Máster en Digital Business. Recuperado el Febrero de 2021, de ESIC Business and Marketing School: https://www.esic.edu/master-y-postgrado/masteren-digital-business-mdb\#madrid

14. ESIC Business and Marketing School. (2020). Master en Dirección de Comunicación y Publicidad. Recuperado el Febrero de 2021, de ESIC Business and Marketing School: https://www.esic.edu/ 
master-y-postgrado/master-en-direccion-de-comunicacion-y-publicidad-mpc

15. ESIC Business and Marketing School. (2020). Master en Dirección de Marketing Deportivo. Recuperado el Febrero de 2021, de ESIC Business and Marketing School: https://www.esic.edu/ master-y-postgrado/master-en-direccion-de-marketing-deportivo-mdmd

16. ESIC Business and Marketing School. (2020). Máster Internacional en Marketing de Moda y Lujo. Recuperado el Febrero de 2021, de ESIC Business and Marketing School: https://www.esic.edu/ master-y-postgrado/master-internacional-en-marketing-de-moda-y-lujo-mml

17. ESPAE Escuela de negocios. (2021). Maestría en Administración y Dirección de Empresas con Mención en Innovación - EMBA. Recuperado el Febrero de 2021, de ESPAE Escuela de negocios: https://www.espae.espol.edu.ec/emba/

18. Garcés León, D., Millán Pelayo, H., Peña Pulido, M., \& Torres Belalcázar, Á. (2020). Estudio descriptivo sobre la percepción de los estudiantes de últimos semestres de mercadeo en modalidad virtual de la Universidad Ean, sobre los cambios producidos por la Industria 4.0 en la mercadotecnia en Colombia(Tesis de especialización). Bogóta .

19. Hernández Sampieri , R., Fernández Collado , C., \& Baptista Lucio, P. (2014). Metodología de la Investigación. McGraw Hill Interamericana editores, S.A de C.V.

20. HULT International Business School. (2017). Global one year MBA. Recuperado el Febrero de 2021, de HULT International Business School: https://www.hult.edu/en/programs/mba/one-year-mba/

21. Ie Business school. (2021). Executive MBA presencial. Recuperado el Febrero de 2021, de Ie Business school: https://www.ie.edu/es/business-school/programas/mba/executive-mba-presencial/

22. Ie Business school. (2021). Global Online MBA. Recuperado el Febrero de 2021, de Ie Business school: https://www.ie.edu/business-school/programs/mba/global-online-mba/

23. IEDE Business School Universidad Andrés Bello. (s.f.). Máster Universitario en Marketing y Comunicación. Recuperado el Febrero de 2021, de IEDE Business School Universidad Andrés Bello: https://www.iede.cl/master-direccion-comercial-marketing-estrategico/\#Plandeestudios

24. IESA Instituto de Estudios Superiores de Administración Escuela de Gerencia. (s.f.). Maestría en Mercadeo. Obtenido de IESA Instituto de Estudios Superiores de Administración Escuela de Gerencia: http://www.iesa.edu.ve/postgrados/maestria-en-mercadeo

25. INCAE Business School. (s.f.). Programas MBA Global. Recuperado el Febrero de 2021, de INCAE Business School: https://www.incae.edu/es/programas-maestria/global-mba.html

26. ITAM Instituto Tecnológico Autónomo de México. (s.f.). MBA Maestría en Administración. Recuperado el Febrero de 2021, de ITAM Instituto Tecnológico Autónomo de México: https://mba. itam.mx/modalidad/MBA_tiempo_parcial\#program

27. ITESO, Universidad Jesuita de Guadalajara. (s.f.). Ganadores de la Beca al Mérito de la Maestría en Administración Master of Business Administration (MBA). Recuperado el Febrero de 2021, de ITESO, Universidad Jesuita de Guadalajara: https:/posgrados.iteso.mx/maestria-administracionmaster-business-administration-mba

28. Jalil Naji, M. (2018). Industria 4.0, competencia digital y el nuevo Sistema de Formación Profesional para el empleo. Revista Internacional y Comparada de relaciones laborales y derecho del empleo, 6(1), 165-194.

29. Lloyd, M. (2020). Desigualdades educativas y la brecha digital en tiempos de COVID-19. En IISUE, Educación y Pandemia una visión académica (págs. 115-116). México: UNAM.

30. Pontificia Universidad Católica De Chile. (s.f.). MBA Executive. Recuperado el Febrero de 2021, de Pontificia Universidad Católica De Chile: https://escueladeadministracion.uc.cl/mba/versiones/ mba-executive/

31. Secretaría de Educación Pública SEP . (2020). Programa sectorial derivado del plan nacional de desarrollo 2019-2024. México: Diario Oficial.

32. UFM Univerdidad Francisco Marroquín Escuela de Negocios. (s.f.). Información General MBA. Recuperado el Febrero de 2021, de UFM Univerdidad Francisco Marroquín Escuela de Negocios: https://en.ufm.edu/maestrias/mba/informacion-general/

33. Universidad Adolfo Ibáñez. (s.f.). Maestría en Dirección de Marketing. Recuperado el Febrero de 2021, de Universidad Adolfo Ibáñez: https://negocios.uai.cl/magister/magister-en-direccion-demarketing/

34. Universidad Adolfo Ibañez. (s.f.). Magister en Marketing. Recuperado el Febrero de 2021, de Universidad Adolfo Ibañez: https://negocios.uai.cl/master-of-science/magister-en-marketing/

35. Universidad de Chile. (2019). Magister en Marketing. Recuperado el Febrero de 2021, de Posgrado Economia y Negocios Universidad de Chile: https://www.postgradouchile.cl/programa/magister/ magister-en-marketing/ 
36. Universidad de Chile. (s.f.). Magíster en Administración (MBA) sin mención y con menciones en Gestión en Finanzas, Gestión en Marketing y Gestión en Recursos Humanos. Recuperado el Febrero de 2021, de Universidad de Chile: https://www.uchile.cl/postgrados/65233/administracion-mbaweekend

37. Universidad de Chile. (s.f.). MBA Uchile full time. Recuperado el Febrero de 2021, de Universidad de Chile: https://www.mbauchile.cl/mba-full-time/

38. Universidad de Chile Postgrado Economía y Negocios. (s.f.). Magíster en Marketing. Recuperado el Febrero de 2021, de Universidad de Chile: https://www.postgradouchile.cl/programa/magister/ magister-en-marketing/

39. Universidad de Ciencias Empresariales y Sociales. (s.f.). Maestría en Marketing Estratégico. Recuperado el Febrero de 2021, de Universidad de Ciencias Empresariales y Sociales: https://www. uces.edu.ar/carreras-posgrados/ciencias-de-la-administracion-comunicacion-y-negocios/maestriamarketing-estrategico

40. Universidad de los Andes de Colombia. (s.f.). Maestría en Mercadeo. Recuperado el Febrero de 2021, de Universidad de los Andes de Colombia: https://administracion.uniandes.edu.co/programas/ maestrias/mercadeo

41. Universidad de San Andrés. (s.f.). Maestría en Marketing y Comunicación. Recuperado el Febrero de 2021, de Universidad de San Andrés: https://udesa.edu.ar/escuela-de-negocios/maestria-enmarketing-y-comunicacion

42. Universidad ESAN. (2019). Maestría en Marketing. Recuperado el Febrero de 2021, de Universidad ESAN: https://www.esan.edu.pe/maestrias/marketing/

43. Universidad Finis Terrae. (2021). Magister en Marketing Aplicado. Recuperado el Febrero de 2021, de Universidad Finis Terrae: https://postgrados.uft.cl/programas/facultad-de-economia-y-negocios/ magister-en-marketing-aplicado/\#

44. Universidad Internacional Del Ecuador. (2020). Maestría en Mercadotecnia con Mención en Estrategia Digital. Recuperado el Febrero de 2021, de Universidad Internacional Del Ecuador: http://www.uide.edu.ec/posgrados-en-linea/maestria-en-mercadotecnia-con-mencion-en-estrategiadigital/?utm_medium=search\&utm_campaign=Maestria\%20\#Objetivos

45. Universidad Nacional de San Antonio Abad de Cusco. (s.f.). Maestría en Marketing Social y Comunicación Para el Desarrollo. Recuperado el Febrero de 2021, de Universidad Nacional de San Antonio Abad de Cusco: http://postgrado.unsaac.edu.pe/maestrias/ma_marketing.php

46. Universidad Nacional Mayor de San Marcos Universidad del Perú, Decana de América. (s.f.). Maestría en Administración en Marketing. Recuperado el Febrero de 2021, de Universidad Nacional Mayor de San Marcos Universidad del Perú, Decana de América: https://www.unmsm.edu.pe/

47. Universidad ORT Uruguay. (s.f.). Master en Administracón de Empresas MBA. Recuperado el Febrero de 2021, de Universidad ORT Uruguay: https://facs.ort.edu.uy/escuela-de-postgrados/mbamaster-en-administracion-de-empresas/plan-de-estudios

48. Universidad Peruana de Ciencias Aplicadas. (s.f.). Maestría en Dirección de Marketing y Gestión Comercial. Recuperado el Febrero de 2021, de Universidad Peruana de Ciencias Aplicadas: https:// postgrado.upc.edu.pe/maestrias-especializadas/maestria-en-direccion-de-marketing-y-gestioncomercial

49. Universidad Privada del Norte. (2019). Maestría en Gerencia de Marketing y Gestión Comercial. Recuperado el Febrero de 2021, de Universidad Privada del Norte: https://postgrado.upn.edu.pe/es/ maestrias/gerencia-marketing-gestion-comercial

50. World Economic Forum. (2019). The Global Competitiveness Report. Recuperado el Febrero de 2021, de World Economic Forum: http://www3.weforum.org/docs/WEF_ TheGlobalCompetitivenessReport2019.pdf 